\title{
Identities Derived from Noncrossing Partitions of Type $B$
}

\author{
William Y. C. Chen ${ }^{1}$, Andrew Y. Z. Wang ${ }^{2}$ and Alina F. Y. Zhao ${ }^{3}$ \\ Center for Combinatorics, LPMC-TJKLC \\ Nankai University, Tianjin 300071, P. R. China \\ ${ }^{1}$ chen@nankai.edu.cn, ${ }^{2}$ yezhouwang@mail.nankai.edu.cn, ${ }^{3}$ zfeiyan@cf c.nankai.edu.cn
}

Submitted: Aug 17, 2009; Accepted: Jun 1, 2011; Published: Jun 14, 2011

Mathematics Subject Classifications: 05A15, 05A19

\begin{abstract}
Based on weighted noncrossing partitions of type $B$, we obtain type $B$ analogues of Coker's identities on the Narayana polynomials. A parity reversing involution is given for the alternating sum of Narayana numbers of type $B$. Moreover, we find type $B$ analogues of the refinements of Coker's identities due to Chen, Deutsch and Elizalde. By combinatorial constructions, we provide type $B$ analogues of three identities of Mansour and Sun also on the Narayana polynomials.
\end{abstract}

\section{Introduction}

The objective of this paper is to give type $B$ analogues of combinatorial identities on the Narayana polynomials $[4,9,10,21,22]$

$$
N_{n}(x)=\sum_{k=1}^{n} N_{n, k} x^{k}, n \geq 1,
$$

where

$$
N_{n, k}=\frac{1}{n}\left(\begin{array}{c}
n \\
k-1
\end{array}\right)\left(\begin{array}{l}
n \\
k
\end{array}\right)
$$

are the Narayana numbers. Let

$$
C_{n}=\frac{1}{n+1}\left(\begin{array}{c}
2 n \\
n
\end{array}\right)
$$


be the $n$-th Catalan number. Using generating functions, Coker $[9,10]$ has derived the following identities

$$
\begin{gathered}
\sum_{k=0}^{n-1} \frac{1}{n}\left(\begin{array}{l}
n \\
k
\end{array}\right)\left(\begin{array}{c}
n \\
k+1
\end{array}\right) x^{k}=\sum_{k=0}^{\lfloor(n-1) / 2\rfloor} C_{k}\left(\begin{array}{c}
n-1 \\
2 k
\end{array}\right) x^{k}(1+x)^{n-2 k-1}, \\
\sum_{k=0}^{n-1} \frac{1}{n}\left(\begin{array}{l}
n \\
k
\end{array}\right)\left(\begin{array}{c}
n \\
k+1
\end{array}\right) x^{2 k}(1+x)^{2(n-1-k)}=\sum_{k=0}^{n-1} C_{k+1}\left(\begin{array}{c}
n-1 \\
k
\end{array}\right) x^{k}(1+x)^{k} .
\end{gathered}
$$

Chen, Yan and Yang [9] have given combinatorial interpretations of these two identities based on weighted Dyck paths and 2-Motzkin paths in answer to a question raised by Coker. It should be noticed that identity (1.1) can also be derived from the following identity due to Simion and Ullman [19], see also Chen, Deng and Du [5]:

$$
\frac{1}{n}\left(\begin{array}{l}
n \\
k
\end{array}\right)\left(\begin{array}{c}
n \\
k+1
\end{array}\right)=\sum_{r=0}^{k-1}\left(\begin{array}{c}
n-1 \\
2 r
\end{array}\right)\left(\begin{array}{c}
n-2 r-1 \\
k-1-r
\end{array}\right) C_{r} .
$$

Recently, Mansour and Sun [16] have established the following three identities for the Narayana polynomials and have given both algebraic and combinatorial proofs:

$$
\begin{aligned}
x^{\frac{n}{2}+1} C_{\frac{n}{2}} & =\sum_{k=0}^{n}(-1)^{n-k}\left(\begin{array}{l}
n \\
k
\end{array}\right) N_{k+1}(x)(1+x)^{n-k}, \\
x^{n+2} C_{n+1} & =\sum_{k=0}^{n}(-1)^{n-k}\left(\begin{array}{l}
n \\
k
\end{array}\right) N_{k+1}\left(x^{2}\right)(1-x)^{2(n-k)}, \\
C_{n} & =\sum_{k=0}^{n} \frac{2 k+1}{2 n+1}\left(\begin{array}{c}
2 n+1 \\
n-k
\end{array}\right) N_{k}(x)(1-x)^{n-k},
\end{aligned}
$$

where $C_{\frac{n}{2}}$ is set to zero if $n$ is odd.

We obtain type $B$ analogues of the above identities of Coker, and Mansour and Sun, based on the structure of type $B$ noncrossing partitions. Recall that a type $B$ partition of $[n]$ is a partition of the set $\{1,2, \ldots, n,-1,-2, \ldots,-n\}$, which may have a unique block called the zero block in which $i$ and $-i$ appear in pairs, such that for any block $B$ of $\pi$, the set $-B$, obtained by negating the elements of $B$, is also a block of $\pi$, see, for example, $[2,3,17,20]$. Evidently, the zero block is a union of antipodal pairs $\{i,-i\}$ if it exists. Moreover, there does not exist any other block $B$ such that $B=-B$. By a pure block we mean a block that contains only positive elements or only negative elements. A block is called a mixed block if it is not pure. A type $B$ partition $\pi$ can be represented by a diagram, with the elements $1,2, \ldots, n,-1,-2, \ldots,-n$ drawn on a horizontal line in the following order

$$
1<2<\cdots<n<-1<-2<\cdots<-n \text {. }
$$


Accordingly, one can list the elements of a block in the above order. Suppose that $B=$ $\left\{i_{1}, i_{2}, \ldots, i_{k}\right\}$ is a nonzero block of a type $B$ partition $\pi$. One may represent this block $B$ by a path from $i_{1}$ to $i_{k}$ with arcs drawn above the horizontal line from $i_{1}$ to $i_{2}, i_{2}$ to $i_{3}$, and so on. A block with one element is called a singleton block. Such a diagram is called the linear representation of a type $B$ partition. A type $B$ partition is said to be noncrossing if its diagram contains no crossing arcs, see, Athanasiadis [3]. It is worth mentioning that we may also place the elements $1,2, \ldots, n,-1,-2, \ldots,-n$ on a circle, and use a cycle to represent a block. This is called the cyclic representation of a type $B$ partition. This leads to an equivalent definition of noncrossing partitions of type $B$, see, Reiner [17]. An illustration of these two representations of a type $B$ noncrossing partition is given in Figure 1.1, where

$$
\pi=\{1,-7\}\{7,-1\}\{2,4,-6\}\{6,-2,-4\}\{3\}\{-3\}\{5,-5\}\{8\}\{-8\} .
$$
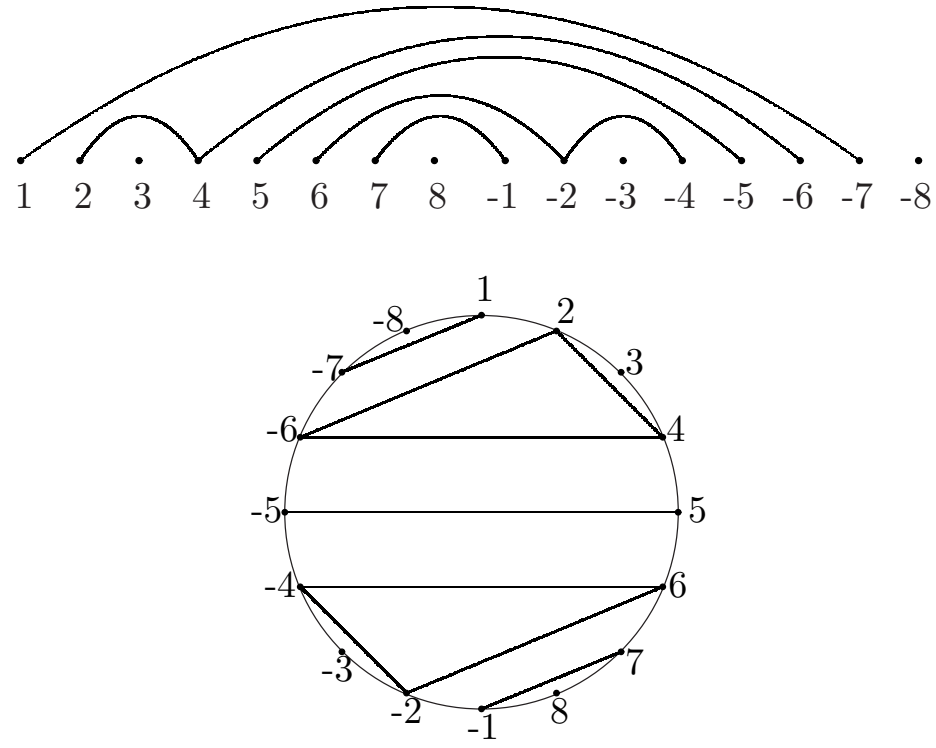

Figure 1.1: The linear and cyclic representations of a type $B$ noncrossing partition.

In this paper, we adopt the linear representation of type $B$ noncrossing partitions. The set of type $B$ noncrossing partitions of $[n]$ will be denoted by $N C^{B}(n)$. It is well known that the cardinality of $N C^{B}(n)$ equals $\left(\begin{array}{c}2 n \\ n\end{array}\right)$, see, for example, Reiner [17, Proposition 6]. Furthermore, the set of type $B$ noncrossing partitions of $[n]$ having $k$ pairs of nonzero blocks will be denoted by $N C^{B}(n, k)$. The cardinality of $N C^{B}(n, k)$, which is known to be $\left(\begin{array}{l}n \\ k\end{array}\right)^{2}$, has been referred to as the Narayana number of type $B$ by Fomin and Reading [12]. The polynomials

$$
P_{n}(x)=\sum_{k=0}^{n}\left(\begin{array}{l}
n \\
k
\end{array}\right)^{2} x^{k}, n \geq 1,
$$

will be called the Narayana polynomials of type $B$. 
This paper is organized as follows. In Section 2, we give type $B$ analogues of Coker's identities and combinatorial proofs in terms of weighted type $B$ noncrossing partitions. We find an involution for the evaluation of the alternating sum of the Narayana numbers of type $B$. We also provide refinements of Coker's identities of type $B$. Section 3 is devoted to type $B$ analogues of three identities due to Mansour and Sun [16].

\section{Type $B$ Analogues of Coker's Identities}

This section is concerned with type $B$ analogues of Coker's identities. We shall use weighted type $B$ noncrossing partitions as the underlying combinatorial structure. The following two identities can be regarded as type $B$ analogues of Coker's identities.

Theorem 2.1. For $n \geq 0$,

$$
\begin{gathered}
\sum_{k=0}^{n}\left(\begin{array}{l}
n \\
k
\end{array}\right)^{2} x^{k}=\sum_{k=0}^{\left\lfloor\frac{n}{2}\right\rfloor}\left(\begin{array}{c}
n \\
2 k
\end{array}\right)\left(\begin{array}{c}
2 k \\
k
\end{array}\right) x^{k}(1+x)^{n-2 k}, \\
\sum_{k=0}^{n}\left(\begin{array}{c}
n \\
k
\end{array}\right)^{2} x^{2 k}(1+x)^{2(n-k)}=\sum_{k=0}^{n}\left(\begin{array}{c}
n \\
k
\end{array}\right)\left(\begin{array}{c}
2 k \\
k
\end{array}\right) x^{k}(1+x)^{k} .
\end{gathered}
$$

Note that identity (2.1) was first derived by Riordan [18] by using generating functions. Before presenting the combinatorial proof of the above theorem, we present a property of the linear representation of a type $B$ noncrossing partition. The proof is straightforward and hence is omitted.

Proposition 2.2. Let $\pi$ be a type $B$ noncrossing partition. In the linear representation of $\pi$, for any pair of antipodal blocks $B$ and $-B$, either one lies entirely on the left of the other, or one is completely covered (or nested) by an arc of the other.

In light of the above property, for a pair of antipodal blocks $B$ and $-B$, we need only one of them to represent this pair. We shall choose the one that is on the left of the other or the one that is nested by an arc of the other. Moreover, we shall list the representative blocks $B_{1}, B_{2}, \ldots, B_{k}$ in the increasing order of their minimum elements. In particular, we use $B_{0}$ to denote the set of positive elements of the zero block. We shall call $B_{0} / B_{1} / B_{2} / \cdots / B_{k}$ the canonical representation of $\pi$. It is clear that the elements appearing in the canonical representation of a type $B$ partition of $[n]$ form a set such that for any $i \in[n]$, either $i$ or $-i$ appears once, but not both. For example, the representative blocks of the noncrossing partition $\pi$ in Figure 1.1 are $B_{0}=\{5\}, B_{1}=\{3\}, B_{2}=\{6,-2,-4\}, B_{3}=\{7,-1\}$ and $B_{4}=\{8\}$.

From now on, we shall use the above canonical representation $\pi=B_{0} / B_{1} / \cdots / B_{k}$ for a type $B$ noncrossing partition. The elements appearing in the canonical representation, namely, the elements of $B_{0}, B_{1}, \ldots, B_{k}$, will be classified into five types. Let $i \in B_{j}$. Then we say that 
1. $i$ is a zero point if $j=0$, that is, $i \in B_{0}$; Otherwise,

2. $i$ is a singleton if $\left|B_{j}\right|=1$;

3. $i$ is a transient point if $i$ is neither the smallest nor the largest element of $B_{j}$, according to the order (1.6);

4. $i$ is a departure point if $i$ is the smallest element of $B_{j}$ and $\left|B_{j}\right|>1$;

5. $i$ is a destination point if $i$ is the largest element of $B_{j}$ and $\left|B_{j}\right|>1$.

For the type $B$ partition $\pi$ in Figure 1.1, 5 is a zero point; 3 and 8 are singletons; -2 is a transient point; 6 and 7 are departure points; -1 and -4 are destination points. Before proving Theorem 2.1, we first present a formula that will be used later.

Proposition 2.3. The number of partitions in $N C^{B}(n)$ with exactly $k$ pairs of nonzero blocks but no singletons equals to

$$
\left(\begin{array}{c}
n \\
2 k
\end{array}\right)\left(\begin{array}{c}
2 k \\
k
\end{array}\right)
$$

Proof. From the correspondence of Reiner [17] between type $B$ noncrossing partitions and pairs $(L, R)$ of $k$-subsets of $[n]$, it is not hard to see that a point $i$ is a singleton of $\pi$ if and only if $i$ appears in both $L$ and $R$. Thus a type $B$ noncrossing partition $\pi$ without singletons is uniquely determined by a pair $(L, R)$ of disjoint subsets of $[n]$ with equal cardinality, namely $L, R \subseteq[n], L \cap R=\emptyset$ and $|L|=|R|$. Moreover, the number of pairs of nonzero blocks equals the cardinality of $|L|$ and $|R|$. Clearly, there are $\left(\begin{array}{c}n \\ 2 k\end{array}\right)\left(\begin{array}{c}2 k \\ k\end{array}\right)$ ways to choose $(L, R)$. This completes the proof.

To make this paper self-contained, we give a more detailed description of the procedure to generate a type $B$ noncrossing partition $\pi$ without singletons from a pair $(L, R)$ of disjoint $k$-subsets of $[n]$ by using the beautiful construction of Reiner [17]. First, put $2 n$ points on a horizontal line with labels $1,2, \ldots, n,-1,-2, \ldots,-n$ from left to right in accordance with the order (1.6). If $l_{i} \in L$, we replace each of the points $l_{i}$ and $-l_{i}$ by a left parenthesis; if $r_{i} \in R$, replace each of the points $r_{i}$ and $-r_{i}$ by a right parenthesis. Thus at the positions of the elements $1,2, \ldots, n,-1,-2, \ldots,-n$, the pair $(L, R)$ corresponds to a sequence of $2 k$ left parentheses, $2 k$ right parentheses and $2 n-4 k$ points.

It is important to recall a property of the $2 k$ parentheses in the positions $1,2, \ldots n$, as discovered by Greene and Kleitman [13] in the construction of a symmetric chain decomposition of the poset of subsets of a finite set. To be more specific, any sequence of left parentheses and right parentheses consists of well-parenthesized segments separated by parentheses which can be read from left to right as $) \cdots)(\cdots($. In other words, the unpaired right parentheses appear before the unpaired left parentheses. Thus any sequence of parentheses can be decomposed into well-parenthesized segments, separated by a sequence of right parentheses followed by left parentheses. For example, the sequence )$(()))(()$ ( has the following decomposition into well-parenthesized segments. 


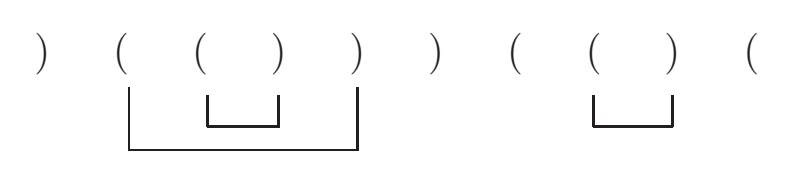

For the well-paired segments at the positive positions, or at the negative positions, we can easily construct pure blocks. For a pair of the form $(\cdots)$, that is, a pair of parentheses for which there are no parentheses between them, we simply form a pure block by selecting the corresponding elements of the parentheses along with the points between them. After such a block is formed, we may delete the underlying elements and continue the above procedure until all well-paired parentheses at the positive positions or at the negative positions are processed.

Upon the deletion of the elements of all pure blocks, the remaining parentheses have the following form

$$
\underbrace{\cdots) \cdots) \cdots) \cdots(\cdots(\cdots(\cdots}_{+} \mid \underbrace{\cdots) \cdots) \cdots) \cdots(\cdots(\cdots(\cdots)}_{-} .
$$

The rightmost left parenthesis at the positive position and the leftmost right parenthesis at the negative position can be well-paired, leading to a mixed block. Once a mixed block $B$ is produced, its negative block $-B$ is determined. It can be shown that a mixed block $B$ formed in the above procedure must be nested by its antipodal block. If $(i,-j)$ is a paired parentheses which yields a mixed block $B$, then $j$ is the largest positive element of $-B$ and $-i$ is the smallest negative element of $-B$. Clearly, the mixed block $B$ is nested by the arc $(j,-i)$ of $-B$ since $j<i$. Moreover, one readily sees that the block $B$ forms a consecutive segment with respect to the order (1.6) after removing the pure blocks, whereas the block $-B$ occupies two consecutive segments at both ends. Then we delete the elements of the antipodal blocks $B$ and $-B$ and iterate this process to get all the noncrossing mixed blocks. When all the pure and mixed blocks are obtained, if there are still some elements left, we collect them together to form the zero block.

Conversely, given a type $B$ noncrossing partition, the pair of subsets $(L, R)$ can be easily determined by the absolute values of the departure points and the destination points. Thus we have obtained the desired bijection. An illustration of this correspondence is given in Figure 2.1.

Combinatorial Proof of (2.1). We shall assign a weight to each point, and the weight of a partition is the product of the weights of all the points. More precisely, we shall assign weights only to half of the elements appearing in the canonical representation of a type $B$ noncrossing partition. The departure points and singletons are assigned weight $x$, the other points (in the canonical representation) are assigned weight 1 . Thus the left-hand side of (2.1) equals the total weight of the set $N C^{B}(n)$.

To give a combinatorial interpretation of the right-hand side, let $S_{k}$ denote the set of partitions in $N C^{B}(n)$ with exactly $k$ pairs of nonzero blocks but with no singletons, and let $T_{k}$ denote the set of partitions in $N C^{B}(n)$ with exactly $k$ pairs of nonzero blocks which have at leat two elements. Clearly, every nonzero block of a partition in $S_{k}$ contains at 


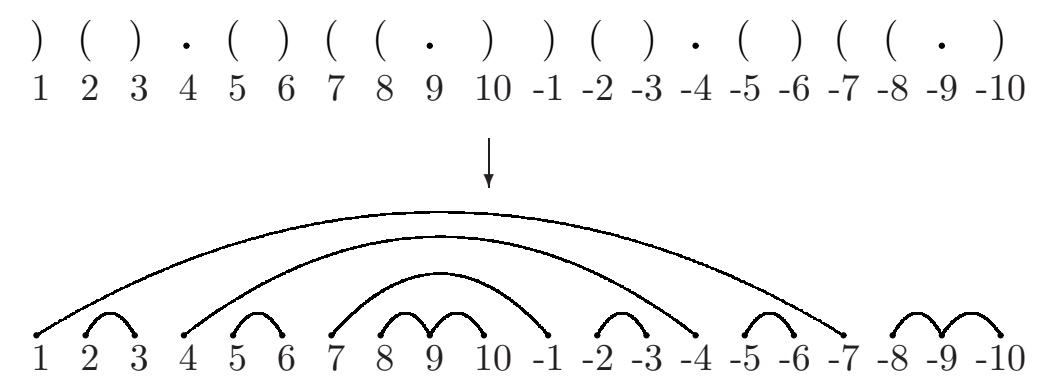

Figure 2.1: The correspondence between a pair $(L, R)$ and a noncrossing partition.

least two elements. By Proposition 2.3, the total weight of $S_{k}$ equals $\left(\begin{array}{c}n \\ 2 k\end{array}\right)\left(\begin{array}{c}2 k \\ k\end{array}\right) x^{k}$. From the above description of the construction for Proposition 2.3, it is clearly seen that a zero point (in the canonical representation) can not be covered by an arc of a nonzero block. Furthermore, a partition in $T_{k}$ can be obtained by changing some zero points and transient points to singletons. Conversely, given a partition in $T_{k}$, there is only one way to change it back to a partition in $S_{k}$ by switching every singleton to either a zero point or a transient point. This implies that the total weight of $T_{k}$ equals the total weight of $S_{k}$ multiplied by a factor $(1+x)^{n-2 k}$. This completes the proof.

Our combinatorial interpretation of $(2.2)$ is based on the set $V_{n}$ of colored type $B$ noncrossing partitions, which consists of all type $B$ noncrossing partitions of $[n]$ for which a pair of antipodal singletons may be colored by one of the two colors, say, black and white.

Combinatorial Proof of (2.2). We assign weight $x^{2}$ to departure points and singletons, and assign weight $(1+x)^{2}$ to zero points, transient points, and destination points. Then the left-hand side of (2.2) equals the total weight of the set $N C^{B}(n)$.

As far as the right-hand side is concerned, we need to classify the set $N C^{B}(n)$ as follows. Given a partition $\pi \in N C^{B}(n)$, let $L_{\pi}$ and $R_{\pi}$ denote the sets of departure points and destination points respectively in the canonical representation of $\pi$. Two partitions $\pi$ and $\sigma$ are in the same class if $\left(L_{\pi}, R_{\pi}\right)=\left(L_{\sigma}, R_{\sigma}\right)$. Suppose that $(L, R)$ is a pair of feasible sets of departure points and destination points, namely, there exists $\pi$ such that $(L, R)=\left(L_{\pi}, R_{\pi}\right)$. Let $G(L, R)$ be the set of partitions $\pi \in N C^{B}(n)$ such that $\left(L_{\pi}, R_{\pi}\right)=(L, R)$. We further assume that both $L$ and $R$ contain $k$ elements. Since the departure points and destination points always appear in pairs and $x^{2}(1+x)^{2}=(x(1+x))^{2}$, the above weight assignment is equivalent to the effect of assigning weight $x(1+x)$ to both departure points and destination points. By the same argument in the proof of (2.1), the noncrossing property implies that the other points can be either singletons or nonsingletons (transient points or zero points), we deduce that the total weight of $G(L, R)$ equals

$$
(x(1+x))^{2 k}\left(x^{2}+(1+x)^{2}\right)^{n-2 k} .
$$


We next proceed to give an alternative interpretation of the total weight (2.3) in terms of colored type $B$ noncrossing partitions in $V_{n}$. Assign weight $x(1+x)$ to black singletons, zero points, transient points, departure points, destination points and weight 1 to white singletons. Let us define $H(L, R)$ for $V_{n}$ in the same way as we defined $G(L, R)$, that is, the set of colored type $B$ noncrossing partitions $\pi$ such that $\left(L_{\pi}, R_{\pi}\right)=(L, R)$ and $|L|=|R|=k$. By the weight assignment for the partitions in $V_{n}$, we see that the total weight of $H(L, R)$ equals

$$
(x(1+x))^{2 k}(1+x(1+x)+x(1+x))^{n-2 k},
$$

which coincides with (2.3). This implies that the right-hand side of (2.2) can be reinterpreted in terms of partitions in $V_{n}$. It remains to show that the total weight of $V_{n}$ equals the right-hand side of (2.2). To construct a partition of $V_{n}$ with $n-k$ white singletons, we may choose $n-k$ white singletons from $[n]$ in $\left(\begin{array}{l}n \\ k\end{array}\right)$ ways. Observe that white singletons have weight 1 and the remaining $2 k$ elements can form a type $B$ noncrossing partition with each element (in the canonical representation) having weight $x(1+x)$. Clearly, there are $\left(\begin{array}{c}2 k \\ k\end{array}\right)$ choices of such partitions with weight $x^{k}(1+x)^{k}$. This completes the proof.

Setting $x=1$, identity (2.1) takes the form

$$
\sum_{m=0}^{\left\lfloor\frac{n}{2}\right\rfloor}\left(\begin{array}{c}
n \\
2 k
\end{array}\right)\left(\begin{array}{c}
2 k \\
k
\end{array}\right) 2^{n-2 k}=\left(\begin{array}{c}
2 n \\
n
\end{array}\right)
$$

which can be regarded as a type $B$-analogue of Touchard's formula

$$
\sum_{k=0}^{\lfloor(n-1) / 2\rfloor}\left(\begin{array}{c}
n-1 \\
2 k
\end{array}\right) C_{k} 2^{n-1-2 k}=C_{n}
$$

Simion [20] has given a combinatorial interpretation of (2.5) by means of a symmetric boolean decomposition of the lattice $N C^{B}(n)$ with the refinement order.

When $x=-1$, identity (2.1) becomes

$$
\sum_{k=0}^{n}(-1)^{k}\left(\begin{array}{l}
n \\
k
\end{array}\right)^{2}= \begin{cases}(-1)^{r}\left(\begin{array}{c}
2 r \\
r
\end{array}\right), & \text { if } n=2 r \\
0, & \text { otherwise }\end{cases}
$$

We shall provide an involution as an explanation of identity (2.6). It should be noted that (2.6) is a type $B$ analogue of the following identity on the alternating sum of Narayana numbers,

$$
\sum_{k=0}^{n}(-1)^{k} \frac{1}{n}\left(\begin{array}{c}
n \\
k-1
\end{array}\right)\left(\begin{array}{l}
n \\
k
\end{array}\right)= \begin{cases}(-1)^{r+1} C_{r}, & \text { if } n=2 r+1 \\
0, & \text { otherwise }\end{cases}
$$

The above identity (2.7) was first discovered by Bonin, Shapiro and Simion [4] in their study of Schröder paths. It was also studied by Coker [10], Klazar [14], Eu, Liu and 
Yeh [11]. A combinatorial proof of (2.7) was given by Chen, Shapiro and Yang [8] by using plane trees and 2-Motzkin paths.

In the case of $x=-\frac{1}{2}$, identity (2.2) reduces to

$$
\sum_{k=0}^{n}(-1)^{k}\left(\begin{array}{l}
n \\
k
\end{array}\right)\left(\begin{array}{c}
2 k \\
k
\end{array}\right) 4^{n-k}=\left(\begin{array}{c}
2 n \\
n
\end{array}\right) .
$$

This identity can be found in Riordan [18]. It was derived by means of generating functions.

We now give a combinatorial interpretation of $(2.6)$. Let $N C_{e}^{B}(n)\left(\operatorname{resp} . N C_{o}^{B}(n)\right)$ denote the set of type $B$ noncrossing partitions of $[n]$ into even (resp. odd) pairs of nonzero blocks. We give a parity reversing involution on type $B$ noncrossing partitions which implies the following formulation of (2.6):

$$
\left|N C_{e}^{B}(n)\right|-\left|N C_{o}^{B}(n)\right|= \begin{cases}(-1)^{r}\left(\begin{array}{c}
2 r \\
r
\end{array}\right), & \text { if } n=2 r \\
0, & \text { otherwise. }\end{cases}
$$

Let $A_{n}$ denote the set of type $B$ noncrossing partitions of $[n]$ without the zero block such that every nonzero block contains exactly two elements. Notice that $A_{n}$ is empty if $n$ is odd and

$$
\left|A_{2 n}\right|=\left(\begin{array}{c}
2 n \\
n
\end{array}\right)
$$

Define the parity of a type $B$ noncrossing partition as the parity of the number of pairs of nonzero blocks. Moreover, we define the sign of a partition as -1 if it is odd, and as 1 if it is even.

Theorem 2.4. There is a parity reversing involution $\rho$ on the set $N C^{B}(n) \backslash A_{n}$.

Proof. Let $\pi=B_{0} / B_{1} / \cdots / B_{k} \in N C^{B}(n) \backslash A_{n}$ be the canonical representation of $\pi$. We first list the elements of $B_{0}, B_{1}, \ldots, B_{k}$ in the increasing order of their absolute values. Then we define the critical point $i$ of $\pi$ as the first element in the above order that is neither a departure point nor a destination point, or equivalently, is either a zero point, or a transient point, or a singleton. We now define the map $\rho$ based on the following cases with respect to the critical point:

If the critical point $i$ is a zero point or a transient point, we take the element $i$ out of the block and form a singleton block $\{i\}$.

If the critical point $i$ is a singleton, we need to determine whether $i$ should be put into the zero block or a nonzero block. We consider the arc between $i$ and $-i$ in the linear representation of $\pi$. If this arc does not cross any nonzero block, then we put $i$ into the zero block. Otherwise, there exits at least one arc covering $i$ or $-i$ or both. In this case, we put $i$ into the nonzero block (resp. the negative of the nonzero block) that has an arc covering $i$ (resp. $-i$ ) and there are no arcs of other blocks covered by this arc. 
It is not hard to see that the above map changes the number of blocks by one. Moreover, the critical point remains unchanged under the above map. Thus we deduce that $\rho$ is a parity reversing involution.

Figure 2.2 gives an example of the involution $\rho$, where the italic 1 is the critical point.

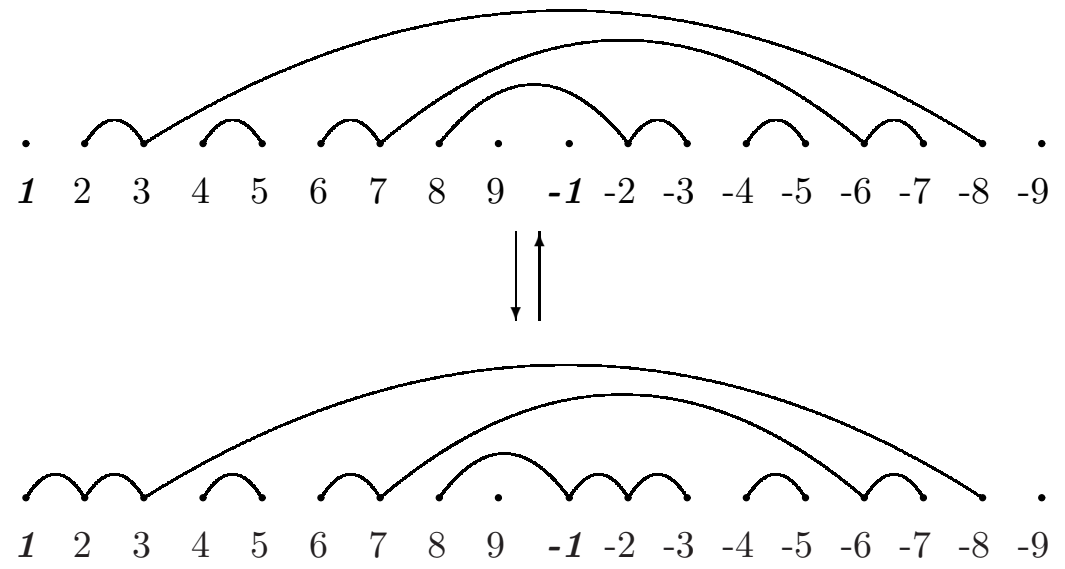

Figure 2.2: The involution $\rho$.

Since $A_{2 n+1}$ is empty and any partition in $A_{2 n}$ has $n$ pairs of nonzero blocks, the involution $\rho$ leads to a combinatorial proof of (2.9).

Appealing to the correspondence between plane trees and 2-Motzkin paths, Chen, Deutsch and Elizalde [6] obtained the following refinements of Coker's identities:

$$
\begin{aligned}
& \sum_{i=1}^{n} \sum_{j=0}^{n-2 i+1} \frac{1}{n}\left(\begin{array}{c}
n \\
i
\end{array}\right)\left(\begin{array}{c}
n-1 \\
j
\end{array}\right)\left(\begin{array}{c}
n-i-j \\
i-1
\end{array}\right) x^{i-1} y^{j}=\sum_{k=0}^{\lfloor(n-1) / 2\rfloor} C_{k}\left(\begin{array}{c}
n-1 \\
2 k
\end{array}\right) x^{k}(1+y)^{n-2 k-1}, \\
& \sum_{i=1}^{n} \sum_{j=0}^{n-2 i+1} \frac{1}{n}\left(\begin{array}{c}
n \\
i
\end{array}\right)\left(\begin{array}{c}
n-1 \\
j
\end{array}\right)\left(\begin{array}{c}
n-i-j \\
i-1
\end{array}\right) x^{2(i-1)} y^{j} z^{n-2 i-j+1}=\sum_{k=0}^{n-1} C_{k+1}\left(\begin{array}{c}
n-1 \\
k
\end{array}\right) x^{k}(y+z-2 x)^{n-1-k} .
\end{aligned}
$$

The following refinements of (2.1) and (2.2) can be viewed as type $B$ analogues of (2.10) and (2.11).

Theorem 2.5. For $n \geq 1$, we have

$$
\begin{gathered}
\sum_{i=0}^{n} \sum_{j=0}^{\left\lfloor\frac{n-i}{2}\right\rfloor}\left(\begin{array}{c}
n \\
i
\end{array}\right)\left(\begin{array}{c}
n-i \\
j
\end{array}\right)\left(\begin{array}{c}
n-j-i \\
j
\end{array}\right) x^{j} y^{i}=\sum_{k=0}^{\left\lfloor\frac{n}{2}\right\rfloor}\left(\begin{array}{c}
n \\
2 k
\end{array}\right)\left(\begin{array}{c}
2 k \\
k
\end{array}\right) x^{k}(1+y)^{n-2 k}, \\
\sum_{i=0}^{n} \sum_{j=0}^{\left\lfloor\frac{n-i}{2}\right\rfloor}\left(\begin{array}{c}
n \\
i
\end{array}\right)\left(\begin{array}{c}
n-i \\
j
\end{array}\right)\left(\begin{array}{c}
n-j-i \\
j
\end{array}\right) x^{2 j} y^{i} z^{n-2 j-i}=\sum_{k=0}^{n}\left(\begin{array}{c}
n \\
k
\end{array}\right)\left(\begin{array}{c}
2 k \\
k
\end{array}\right) x^{k}(y+z-2 x)^{n-k} .
\end{gathered}
$$


Proof. We first consider (2.12) and shall also use weighted type $B$ noncrossing partitions to give the combinatorial interpretations of both sides of (2.12). The weight assignment is almost the same as in the proof of identity (2.1) except that a singleton is endowed with weight $y$. Suppose that $\pi$ is a type $B$ noncrossing partition with $i$ singletons (in the canonical representation, to be precise). The remaining $n-i$ elements form a type $B$ noncrossing partition $\sigma$ such that each nonzero block contains at leat two elements (in the canonical representation as well). Assume that $\sigma$ contains $j$ nonzero blocks, by Proposition 2.3, there are $\left(\begin{array}{c}n-i \\ j\end{array}\right)\left(\begin{array}{c}n-j-i \\ j\end{array}\right)=\left(\begin{array}{c}n-i \\ 2 j\end{array}\right)\left(\begin{array}{c}2 j \\ j\end{array}\right)$ choices for $\sigma$. According to the weight assignment, $\sigma$ has weight $x^{j}$. In view of the number of singletons, we see that the left-hand side of (2.12) equals the sum of the weights of all type $B$ noncrossing partitions of $[n]$.

Regarding the right-hand side, let $S_{k}$ be the set of the partitions in $N C^{B}(n)$ with $k$ pairs of nonzero blocks but with no singletons, and let $T_{k}$ be the set of partitions in $N C^{B}(n)$ with exactly $k$ pairs of nonzero and nonsingleton blocks. The weight of any partition in $S_{k}$ is $x^{k}$. Meanwhile, there are $\left(\begin{array}{c}n \\ 2 k\end{array}\right)\left(\begin{array}{c}2 k \\ k\end{array}\right)$ partitions in $S_{k}$. Using the same argument as in the proof of (2.1), we see that the total weight of $T_{k}$ equals the total weight of $S_{k}$ multiplied by a factor $(1+y)^{n-2 k}$. Thus the right-hand side of $(2.12)$ also equals the total weight of $N C^{B}(n)$. This proves (2.12).

We now proceed to prove (2.13) by using a different weight assignment. Assign weight $x$ to departure points and destination points, weight $y$ to singletons and weight $z$ to zero points, transient points. Suppose that $\pi$ is a partition with $i$ singletons and $j$ nonzero blocks with at least two elements. Since the departure points and destination points appear in pairs, there are $n-2 j-i$ other elements with weight $z$. From Proposition 2.3 it follows that the left-hand side of (2.13) equals the total weight of the set $N C^{B}(n)$.

On the other hand, we may consider the set $V_{n}$ of colored type $B$ noncrossing partitions, as defined before. Assign weight $x$ to black singletons, zero points, transient points, departure points, destination points, and assign weight $y+z-2 x$ to white singletons. Let $G(L, R)$ and $H(L, R)$ denote the subsets of $N C^{B}(n)$ and $V_{n}$ respectively as in the proof of identity (2.2). According to the weight assignment, the departure points and destination points have weight $x$, singletons have weight $y$ and the other points have weight $z$, thus the total weight of the set $G(L, R)$ is $x^{2 k}(y+z)^{n-2 k}$. Similarly, the total weight of $H(L, R)$ equals $x^{2 k}(x+x+y+z-2 x)^{n-2 k}$, which coincides with the total weight of $G(L, R)$.

Now it suffices to show that the total weight of $V_{n}$ agrees with the right-hand side of (2.2). In order to compute the total weight of $V_{n}$, we can first choose $n-k$ white singletons from $[n]$ in $\left(\begin{array}{l}n \\ k\end{array}\right)$ ways. These white singletons have weight $(y+z-2 x)^{n-k}$. The remaining $2 k$ elements form a type $B$ noncrossing partition with each point having weight

$x$. There are $\left(\begin{array}{c}2 k \\ k\end{array}\right)$ choices of such partitions and the weight of such a partition equals $x^{k}$. This completes the proof. 


\section{Type $B$ Analogues of the Identities of Mansour and Sun}

In this section, we provide type $B$ analogues along with combinatorial proofs of the identities (1.3), (1.4) and (1.5) due to Mansour and Sun [16]. We begin with the following identity which can be considered as a type $B$ analogue of (1.3).

Theorem 3.1. For $n \geq 0$, we have

$$
\sum_{k=0}^{n}(-1)^{n-k}\left(\begin{array}{l}
n \\
k
\end{array}\right) P_{k}(x)(1+x)^{n-k}=\left\{\begin{array}{cl}
x^{r}\left(\begin{array}{c}
2 r \\
r
\end{array}\right), & \text { if } n=2 r \\
0, & \text { otherwise. }
\end{array}\right.
$$

Setting $x=1$, (3.1) reduces to the following identity of Dawson, see, Riordan [18, p.71],

$$
\sum_{k=0}^{n}(-1)^{n-k}\left(\begin{array}{l}
n \\
k
\end{array}\right)\left(\begin{array}{c}
2 k \\
k
\end{array}\right) 2^{n-k}=\left\{\begin{array}{cc}
\left(\begin{array}{c}
2 r \\
r
\end{array}\right), & \text { if } n=2 r \\
0, & \text { otherwise. }
\end{array}\right.
$$

Andrews [1] has given a proof of (3.2) by employing Gauss's second summation theorem, which states that

$$
{ }_{2} F_{1}\left[\begin{array}{c}
a, b \\
1 / 2+a / 2+b / 2
\end{array} ; 1 / 2\right]=\frac{\Gamma(1 / 2) \Gamma(1 / 2+a / 2+b / 2)}{\Gamma(1 / 2+a / 2) \Gamma(1 / 2+b / 2)} .
$$

Combinatorial Proof of Theorem 3.1. The left-hand side of (3.1) can be interpreted in terms of weighted type $B$ colored partitions in $V_{n}$. We use the following weight assignment. Transient points, zero points and destination points are given weight 1 ; black singletons and departure points are given weight $x$; white singletons are given weight $-1-x$.

To construct a partition in $V_{n}$, we first choose a subset $S$ of $n-k$ elements from $[n]$ to form white singletons with weight $-1-x$. There are $\left(\begin{array}{l}n \\ k\end{array}\right)$ ways to choose $S$, and these white singletons will contribute a factor $(-1-x)^{n-k}$ to the weight. On the other hand, the remaining elements constitute a noncrossing partition of $2 k$ elements with the same weight assignment except that the singletons are assumed to be black. It follows that such partitions have total weight $P_{k}(x)$. Thus the left-hand side of (3.1) equals the total weight of the set $V_{n}$.

We now aim to construct a sign reversing involution $\theta$ on $V_{n} \backslash A_{n}$, where $A_{n}$ is defined in the preceding section. By the definition of $A_{n}$, we may regard the partitions in $A_{n}$ as noncrossing partitions in $V_{n}$ with no zero block, no singletons and no transient points. The involution $\theta$ can be described as follows.

Given a type $B$ colored noncrossing partition $\pi \in V_{n} \backslash A_{n}$, we define the critical point as the first point $i$ (in the increasing order of the absolute values) in the canonical representation of $\pi$ that is neither a departure point nor a destination point of $\pi$. Then we conduct the following operations: 
- If $i$ is a black singleton, then we change it to a white singleton with weight $-x$;

- If $i$ is a transient point or a zero point, then we change it to a white singleton with weight -1 ;

- If $i$ is a white singleton with weight $-x$, then we change it to a black singleton;

- If $i$ is a white singleton with weight -1 , then we change it to a transient point or a zero point according to whether the arc $(i,-i)$ crosses any arc of nonzero blocks as in the proof of Theorem 2.4.

It is easily seen that the critical cannot be mapped to a departure point or a destination point. Moreover, in the increasing order of the absolutes, the elements before $i$ stay unchanged since the above operations do not cause additional departure points or destination points. Therefore, the critical point remains the same. Thus $\theta$ is a sign reversing and weight preserving involution.

Our final task is to compute the total weight of $A_{n}$. First consider the case when $n$ is odd. For a type $B$ noncrossing partition $\pi$ of $[n]$, it is clear that there exists at least one point which is neither a departure point nor a destination point. By the involution $\theta$, we deduce that the sum of weights of type $B$ colored noncrossing partitions of $[n]$ equals zero. For $n=2 r$, the total weight of $V_{n}$ equals to the total weight of $A_{n}$, which equals $x^{r}\left(\begin{array}{c}2 r \\ r\end{array}\right)$. This completes the proof.

Figure 3.1 is an illustration of the involution $\theta$, where a circle stands for a white singleton.
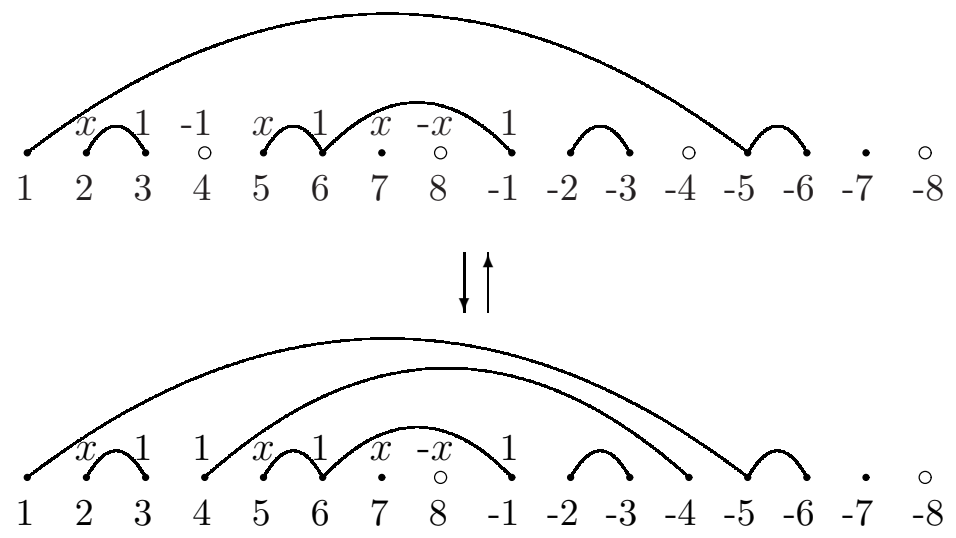

Figure 3.1: The involution $\theta$ on a type $B$ colored noncrossing partition.

We now turn to a type $B$ analogue of identity (1.4).

Theorem 3.2. For $n \geq 0$, we have

$$
\sum_{k=0}^{n}(-1)^{n-k}\left(\begin{array}{l}
n \\
k
\end{array}\right) P_{k}\left(x^{2}\right)(1-x)^{2(n-k)}=x^{n}\left(\begin{array}{c}
2 n \\
n
\end{array}\right) .
$$


It should be noted that setting $x=-1$ in (3.3), we arrive at (2.8) again.

Combinatorial Proof of (3.3). The proof is similar to that of (3.1). We also consider type $B$ colored noncrossing partitions with a different weight assignment. Destination points, zero points and transient points are given weight 1; departure points and black singletons are given weight $x^{2}$; white singletons are given weight $-1+2 x-x^{2}$, or, equivalently, the weight of a white singleton can be either -1 , or $2 x$ or $-x^{2}$. Then the left-hand side of (3.3) can be interpreted as the total weight of the set $V_{n}$.

Let $D_{n}$ be the set of colored noncrossing partitions in $V_{n}$ that have only two types of points (in the canonical representation): (1) departure points or destination points. (2) white singletons with weight $2 x$. In other words, for any partition in $D_{n}$, the following four types of points are not allowed: (1) zero points; (2) transient points; (3) black singletons; (4) white singletons with weight -1 or $-x^{2}$. To prove (3.3), we proceed to construct a sign reversing and weight preserving involution $\eta$ on the set $V_{n} \backslash D_{n}$.

Given a type $B$ colored noncrossing partition $\pi \in V_{n} \backslash D_{n}$, we seek the first point $i$ in the increasing order of the absolute values which is neither a departure point nor a destination point. As usual, $i$ is called the critical point. The map $\eta$ is defined by the following operations:

- If $i$ is a black singleton, then set $i$ to be a white singleton with weight $-x^{2}$;

- If $i$ is a transient point or a zero point, then set $i$ to be a white singleton with weight -1 ;

- If $i$ is a white singleton with weight $-x^{2}$, then set $i$ to be a black singleton;

- If $i$ is a white singleton with weight -1 , then set $i$ to be a transient point or a zero point according to the criterion as given before.

It can be verified that $\eta$ is a sign reversing and weight preserving involution. Thus the total weight of $V_{n}$ equals the total weight of $D_{n}$.

It remains to show that the total weight of the set $D_{n}$ equals the right-hand side of (3.3), namely, $x^{n}\left(\begin{array}{c}2 n \\ n\end{array}\right)$. To construct a weighted type $B$ colored noncrossing partition in $D_{n}$, we may choose $2 k$ elements from $[n]$ to construct a type $B$ noncrossing partition with $k$ departure points and $k$ destination points. There are $\left(\begin{array}{c}n \\ 2 k\end{array}\right)\left(\begin{array}{c}2 k \\ k\end{array}\right)$ choices. The remaining $n-2 k$ points are taken to be white singletons with weight $2 x$. Thus the total weight of the set $D_{n}$ equals

$$
\sum_{k=0}^{\lfloor n / 2\rfloor}\left(\begin{array}{c}
n \\
2 k
\end{array}\right)\left(\begin{array}{c}
2 k \\
k
\end{array}\right) x^{2 k}(2 x)^{n-2 k},
$$

which can be rewritten as

$$
x^{n} \sum_{k=0}^{\lfloor n / 2\rfloor}\left(\begin{array}{c}
n \\
2 k
\end{array}\right)\left(\begin{array}{c}
2 k \\
k
\end{array}\right) 2^{n-2 k} .
$$

Invoking identity (2.5) gives (3.3). This completes the proof. 
We remark in passing that (1.5) is related to the following identity obtained by Chen and Pang [7]

$$
\sum_{k=0}^{n} \frac{1}{n}\left(\begin{array}{c}
n \\
k-1
\end{array}\right)\left(\begin{array}{l}
n \\
k
\end{array}\right) x^{k}(x+1)^{n-k}=\sum_{k=0}^{n}(-1)^{n-k}\left(\begin{array}{c}
n+k \\
n-k
\end{array}\right) \frac{1}{k+1}\left(\begin{array}{c}
2 k \\
k
\end{array}\right)(x+1)^{k},
$$

which was independently derived by Mansour and Sun [15] in a slightly different form

$$
\sum_{k=0}^{n} \frac{1}{n}\left(\begin{array}{c}
n \\
k-1
\end{array}\right)\left(\begin{array}{l}
n \\
k
\end{array}\right) x^{k}=\sum_{k=0}^{n}\left(\begin{array}{c}
n+k \\
n-k
\end{array}\right) \frac{1}{k+1}\left(\begin{array}{c}
2 k \\
k
\end{array}\right)(x-1)^{n-k} .
$$

Upon substituting $x$ with $x /(1-x)$, we see that (3.4) can be recast in the form of $(3.5)$, that is,

$$
\frac{N_{n}(x)}{(x-1)^{n}}=\sum_{k=0}^{n}\left(\begin{array}{l}
n+k \\
n-k
\end{array}\right) C_{k} \frac{1}{(x-1)^{k}} .
$$

Then identity (1.5) follows from the Legendre inversion formula [18]

$$
a_{n}=\sum_{k=0}^{n}\left(\begin{array}{c}
n+k \\
n-k
\end{array}\right) b_{k} \Longleftrightarrow b_{n}=\sum_{k=0}^{n}(-1)^{n-k} \frac{2 k+1}{2 n+1}\left(\begin{array}{c}
2 n+1 \\
n-k
\end{array}\right) a_{k} .
$$

Below is a type $B$ analogue of (3.4), which yields a type $B$ analogue of (1.5).

Theorem 3.3. For $n \geq 0$, we have

$$
\sum_{k=0}^{n}\left(\begin{array}{l}
n \\
k
\end{array}\right)^{2} x^{k}(x-1)^{n-k}=\sum_{k=0}^{n}(-1)^{n-k}\left(\begin{array}{c}
n+k \\
k
\end{array}\right)\left(\begin{array}{l}
n \\
k
\end{array}\right) x^{k} .
$$

Proof. We use weighted type $B$ noncrossing partitions of $[n]$ to give a combinatorial interpretation of the left-hand side. A departure point or a singleton is endowed with weight $x$, whereas the other kinds of points are endowed with weight $x-1$. In this way, the left-hand side of (3.7) equals the total weight of the set $N C^{B}(n)$.

We proceed to show that the summand of the right-hand side equals the sum of weights of type $B$ noncrossing partitions of $[n]$ with exactly $k$ elements having weight $x$. Such partitions can be constructed as follows. We first choose a $k$-subset $S$ of $[n]$ under the condition that if an element $i$ or $-i$ has weight $x$, then $i \in S$. Then we choose $m$ elements from $S$ to be departure points or singletons, denoted by $L=\left\{l_{1}, l_{2}, \ldots, l_{m}\right\}$. Meanwhile, we choose another set of $m$ elements from the set $[n]$, denoted by $R=\left\{r_{1}, r_{2}, \ldots, r_{m}\right\}$. Applying the bijection of Reiner [17] to the pair $(L, R)$, we get a noncrossing partition with $m$ pairs of antipodal nonzero blocks.

We need to compute the sum of weights of such partitions. Note that the departure points and singletons always have weight $x$ and consequently belong to $S$. It suffices to determine which of the other types of points can be given weight $x$. The weight of the 
destination points, zero points and transient points can be either $x$ or -1 subject to the condition for the choice of $S$. If the absolute value of such an element belongs to the set $S$, then it has weight $x$; otherwise, it has weight -1 . Thus, subject to the condition on the choice of $S$, the weight assignment for all the elements are uniquely determined. So the weight of a partition constructed by the above procedure equals $(-1)^{n-k} x^{k}$. On the other hand, the number of such partitions equals

$$
\left(\begin{array}{l}
n \\
k
\end{array}\right) \sum_{m=0}^{k}\left(\begin{array}{l}
k \\
m
\end{array}\right)\left(\begin{array}{l}
n \\
m
\end{array}\right)=\left(\begin{array}{l}
n \\
k
\end{array}\right)\left(\begin{array}{c}
n+k \\
k
\end{array}\right) .
$$

This completes the proof.

In virtue of identity (3.7), we can express the central binomial coefficients in terms of the Narayana polynomials of type $B$.

Theorem 3.4. For $n \geq 0$,

$$
\left(\begin{array}{c}
2 n \\
n
\end{array}\right)=\sum_{k=0}^{n} \frac{2 k+1}{2 n+1}\left(\begin{array}{c}
2 n+1 \\
n-k
\end{array}\right) P_{k}(x)(1-x)^{n-k} .
$$

Proof. Substituting $x$ with $\frac{1}{1-x}$ in (3.7), and observing that

$$
\left(\begin{array}{c}
n+k \\
k
\end{array}\right)\left(\begin{array}{l}
n \\
k
\end{array}\right)=\left(\begin{array}{c}
n+k \\
n-k
\end{array}\right)\left(\begin{array}{c}
2 k \\
k
\end{array}\right)
$$

we find

$$
\sum_{k=0}^{n}\left(\begin{array}{l}
n \\
k
\end{array}\right)^{2} x^{k}=\sum_{k=0}^{n}(-1)^{n-k}\left(\begin{array}{c}
n+k \\
n-k
\end{array}\right)\left(\begin{array}{c}
2 k \\
k
\end{array}\right)(1-x)^{n-k},
$$

which serves as a type $B$ analogue of (3.6). Rewriting (3.9) as

$$
\frac{P_{n}(x)}{(x-1)^{n}}=\sum_{k=0}^{n}\left(\begin{array}{c}
n+k \\
n-k
\end{array}\right)\left(\begin{array}{c}
2 k \\
k
\end{array}\right) \frac{1}{(x-1)^{k}},
$$

applying the Legendre inversion formula, we arrive at (3.8). This completes the proof.

Acknowledgments. The authors wish to thank the referees for helpful comments leading to an improvement of an earlier version. This work was supported by the 973 Project, the PCSIRT Project of the Ministry of Education, and the National Science Foundation of China. 


\section{References}

[1] G. E. Andrews, Applications of basic hypergeometric functions, SIAM Rev., 16 (1974) 441-484.

[2] D. Armstrong, Generalized Noncrossing Partitions and Combinatorics of Coxeter Groups, Ph.D. Thesis, Cornell University, 2006.

[3] C. A. Athanasiadis, On noncrossing and nonnonnesting partitions for classical reflection groups, Electron. J. Combin., 5 (1998) R42.

[4] J. Bonin, L. Shapiro and R. Simion, Some $q$-analogues of the Schröder numbers arising from combinatorial statisics on lattice paths, J. Statist. Plann. Inference., 34 (1993) 35-55.

[5] W. Y. C. Chen, E. Y. P. Deng and R. R. X. Du, Reduction of $m$-regular noncrossing partitions, European J. Combin., 26 (2005) 237-243.

[6] W. Y. C. Chen, E. Deutsch and S. Elizalde, Old and young leaves on plane trees and 2-Motzkin paths, European J. Combin., 27 (2006) 414-427.

[7] W. Y. C. Chen and S. X. M. Pang, On the combinatorics of the Pfaff identity, Discrete Math., 309 (2009) 2190-2196.

[8] W. Y. C. Chen, L. W. Shapiro and L. L. M. Yang, Parity reversing involutions on plane trees and 2-Motzkin paths, European J. Combin., 27 (2006) 283-289.

[9] W. Y. C. Chen, S. H. F. Yan and L. L. M. Yang, Identities from weighted Motzkin paths, Adv. in Appl. Math., 41 (2008) 329-334.

[10] C. Coker, Enumerating a class of lattice paths, Discrete Math., 271 (2003) 13-28.

[11] S.-P. Eu, S.-C. Liu and Y.-N. Yeh, Odd or even on plane trees, Discrete Math., 281 (2004) $189-196$.

[12] S. Fomin and N. Reading, Root systems and generalized associahedra, Lecture notes for the IAS/Park City Graduate Summer School in Geometric Combinatorics, 2004.

[13] C. Greene and D. J. Kleitman, Strong versions of Sperner's Theorem, J. Combin. Theory Ser. A, 20 (1976), 80-88.

[14] M. Klazar, Counting even and odd partitions, Amer. Math. Monthly, 110 (2003) 527-532.

[15] T. Mansour and Y. Sun, Dyck paths and partial Bell polynomials, Australas. J. Combin., 42 (2008) 285-297.

[16] T. Mansour and Y. Sun, Identities involving Narayana polynomials and Catalan numbers, Discrete Math., 309 (2009) 4079-4088.

[17] V. Reiner, Non-crossing partitions for classical reflection groups, Discrete Math., 177 (1997) $195-222$.

[18] J. Riordan, Combinatorial Identities, John Wiley, New York, 1968.

[19] R. Simion and D. Ullman, On the structure of the lattice of noncrossing partitions, Discrete Math., 98 (1991) 193-206.

[20] R. Simion, Combinatorial statistics on type-B analogues of noncrossing partitions and restricted permutations, Electron. J. Combin., 7 (2000) R9.

[21] R. A. Sulanke, Counting lattice paths by Narayana polynomials, Electron. J. Combin., 7 (2000) R40.

[22] R. A. Sulanke, Generalizing Narayana and Schröder numbers to higher dimensions, Electron. J. Combin., 11 (2004) R54. 